\title{
ANU RADIOCARBON DATE LIST XI: RADIOCARBON DATES FROM LAKES BARRINE AND EACHAM, ATHERTON TABLELAND, NORTH QUEENSLAND, AUSTRALIA
}

\author{
M. J. HEAD, L. J. TAYLOR and D. WALKER
}

Quaternary Dating Research Centre, The Australian National University, P. O. Box 4 Canberra, ACT 0200 Australia

\section{INTRODUCTION}

Lakes Barrine and Eacham and their small, discrete, forested catchments are sites of intensive sedimentological, palynological and ecological research. The studies that we present here concentrate on integrating population statistics of tree species of the living forest with their histories, as documented by fine-resolution pollen analysis (Green and Dolman 1988). Most of the work has been undertaken at Lake Barrine, where the last $5 \mathrm{ka}$ is recorded in laminated sediments. Additional information about these sites and about aspects of the project is contained in Chen $(1986,1987,1988)$, Goodfield (1988), Grindrod (1979), Timms $(1976,1979)$ and Walker (1988).

The local bedrock is Cenozoic basalt, and the crater rims are pyroclastic mixtures of basalt and metamorphic fragments. The ages of the craters' formations are not known, but are probably $>1 \mathrm{Ma}$. The rims are covered by wet tropical rainforest. Neither lake has inflowing streams. Barrine overflows through a stream only in summer, but Eacham water seems to be lost by seepage through the crater walls; annual water-level fluctuations do not exceed $40 \mathrm{~cm}$ at either lake. Table 1 shows comparative statistics for the two lakes.

TABLE 1. Comparative Statistics of Lakes Barrine and Eacham

\begin{tabular}{lll}
\hline & Lake Barrine & Lake Eacham \\
\hline Long/Lat & $145^{\circ} 38^{\prime} \mathrm{E}, 17^{\circ} 15^{\prime} \mathrm{S}$ & $145^{\circ} 37^{\prime} \mathrm{E}, 17^{\circ} 17^{\prime} \mathrm{S}$ \\
Altitude (m asl) & 725 & 747 \\
Total catchment $\left(\mathrm{km}^{2}\right)$ & 1.95 & 0.77 \\
Water surface $\left(\mathrm{km}^{2}\right)$ & 1.00 & 0.47 \\
Mean height of rim (m) & 27 & 30 \\
Maximum water depth (m) & 67 & 63 \\
\hline
\end{tabular}

Both lakes stratify for $\sim 4$ months each summer with a thermocline at $\sim 15 \mathrm{~m}$ depth. Hypolimnion temperature is $19-19.5^{\circ} \mathrm{C}$ year round. Deep mixing occurs during some winters, more frequently in Eacham than Barrine. The lake waters are very dilute, $\sim 60 \mathrm{ppm}$ total dissolved solutes, except in the lowest few meters where $100 \mathrm{ppm}$ may occur at maximum stratification. At Barrine, the maximum depth sampled was $12 \mathrm{~m}$ below mud-water interface at the middle of the lake. The gross stratigraphy from $\sim 30$ cores and the generalized ${ }^{14} \mathrm{C}$ chronology are shown in Tables 2 and 3 . At Eacham, the maximum depth sampled was $6 \mathrm{~m}$, at which the ${ }^{14} \mathrm{C}$ age, at the middle of the lake, is $\sim 5000 \mathrm{BP}$. The muds are not so consistently stratified as at Barrine, are more detrital and have higher clay content.

\section{METHODS}

${ }^{14} \mathrm{C}$ determinations were made on samples from 5 -cm-diameter cores collected with a Mackereth sampler. These cores were transported to the laboratory and exposed by cutting the core longitudi- 
nally. Sediments collected by in-situ freezing were transported to the laboratory packed in solid $\mathrm{CO}_{2}$ and stored in freezers until sampled by dissection over a bed of solid $\mathrm{CO}_{2}$. Levels are from the top of the relevant core with allowance for gaps caused by outgassing. The brief characterizations of the dated materials given here will be fully expanded in later publications. We use the terms, "mud", "lake mud" and "organic mud" as synonyms; "lake sediment" implies a more obvious inorganic content. We describe pretreatment methods below for reference. Except where otherwise noted, we used pretreatment $\mathbf{A}$.

Pretreatment $A$. Rootlets and/or other extraneous materials were removed by hand or by washing, the sample was broken into small pieces, boiled in $2 \mathrm{~N} \mathrm{HCl}$, filtered, rinsed in demineralized water to $\mathrm{pH} \sim 6.5$ and dried.

Pretreatment $B(i)$. Rootlets and/or other extraneous materials were removed by hand, washing or sieving. The sample was broken into small pieces or sieved, boiled in dilute $\mathrm{HCl}$, rinsed to $\mathrm{pH} \sim 6.5$ with demineralized water, and then boiled in $2 \% \mathrm{NaOH}$. The sample $(\mathrm{NaOH}$ insoluble) was then acidified, allowed to settle or was centrifuged, filtered, rinsed with demineralized water to $\mathrm{pH} \sim 6.5$ and dried.

Pretreatment $B$ (ii). The supernatant of $\mathrm{B}(\mathrm{i})$ was further treated with $10 \% \mathrm{HCl}$ to give a $\mathrm{pH}$ of $\sim 2$ and briefly boiled. The $\mathrm{NaOH}$ soluble sample (humic acids) was then allowed to settle or was centrifuged. The precipitate was filtered, rinsed with demineralized water to $\mathrm{pH} \sim 5$ and dried.

Ages are reported as ${ }^{14} \mathrm{C} \mathrm{yr} \mathrm{BP,} \mathrm{i.e.,} \mathrm{corrected} \mathrm{for} \mathrm{isotopic} \mathrm{fractionation} \mathrm{and} \mathrm{based} \mathrm{on} \mathrm{the} \mathrm{Libby} \mathrm{half-}$ life of $5568 \mathrm{yr}$. The modern reference standard was ANU sucrose, secondary international calibration standard, correlated with $95 \%$ of ${ }^{14} \mathrm{C}$ activity of NBS oxalic acid, normalized to $\delta^{13} \mathrm{C}=-19 \% 0$ wrt PDB (Polach 1979; Currie and Polach 1980). $\delta^{13} \mathrm{C}$ values were estimated at $-24.0 \%$, and the dilution factor is zero, unless otherwise indicated.

Barrine-Core Samples from Water Depth Presently $>50 m$ (Table 2)

TABLE 2. Barrine Cores (water depth presently $>50 \mathrm{~m}$ )

\begin{tabular}{lll}
\hline Depth $(\mathrm{m})$ & Age (ka BP) & \multicolumn{1}{c}{ Sediment } \\
\hline $0-4.5$ & $0-5$ & Strongly laminated mud, 30\% dry weight organic \\
$4.5-6$ & $5-10$ & Unlaminated mud, 15\% dry weight organic \\
$6 \rightarrow 12$ & $10->18$ & Clay, sand, gravel, sometimes banded \\
\hline
\end{tabular}

\section{Core B6 Series}

Collected by D. Walker and J. L. Neale 1977; submitted by D. Walker 1978.

ANU-2072. Lake mud, $430-560 \mathrm{~cm}$ depth.

ANU-2072A. (660-min count)

$5770 \pm 110$

ANU-2072B. Dilution; $10 \%$ sample (3280-min count).

$4390 \pm 290$

Comment: Fraction A was given pretreatment B(i) following an ultrasound demineralized water wash; fraction $B$ was given pretreatment $B(i i)$.

ANU-2073. Lake mud, $60.5-76.5 \mathrm{~cm}$ depth.

ANU-2073A. Dilution; $56 \%$ sample (700-min count).

$5950 \pm 160$

ANU-2073B. Dilution; 39\% sample (700-min count).

$5660 \pm 200$ 
Comment: Fraction A was given pretreatment B(i) following an ultrasound demineralized water wash; fraction B was given pretreatment B(ii). Depths are only approximate due to slight drying of the core.

\section{Core B8 Series}

Collected by D. Walker and J. L. Neale 1977; submitted by D. Walker 1978.

ANU-2074.

$1800 \pm 170$

Organic mud, $20-30 \mathrm{~cm}$ depth. Dilution; $27 \%$ sample (900-min count).

ANU-2075.

$4360 \pm 100$

Organic mud, 143-154 cm depth. Dilution; 59\% sample (1700-min count).

ANU-2076.

$4640 \pm 260$

Organic mud, $161-171 \mathrm{~cm}$ depth. Dilution; 29\% sample (520-min count).

ANU-2081.

$6330 \pm 170$

Lake mud, 208-218 cm depth. Dilution; 47\% sample (900-min count).

Comment: ANU-2074 to -2076 and -2081 were suspended in demineralized water, acidified to $\mathrm{pH}$ 2 , allowed to stand, filtered, rinsed in demineralized water and dried.

ANU-2082.

$8320 \pm 270$

Lake mud, 323-333 cm depth. Dilution; 34\% sample (900-min count).

ANU-2083.

$13,000 \pm 290$

Lake clay-mud, 557-567 cm depth. Dilution; 55\% sample (1000-min count).

\section{Core B55/78 Series}

Collected by D. Walker and J. L. Neale 1978; submitted by D. Walker 1979.

ANU-3595.

$1080 \pm 80$

Lake mud, $22-42 \mathrm{~cm}$ depth. Dilution; $72 \%$ sample (1080-minute count).

ANU-2255.

Lake mud, 141-161 cm depth. Dilution; 30\% sample (1140-min count).

ANU-2256.

$3420 \pm 90$

Lake mud, $164.5-184.5 \mathrm{~cm}$ depth. Dilution; $73.8 \%$ sample (1160-min count).

ANU-2257.

$4200 \pm 100$

Lake mud, 267-287 cm depth. Dilution; 58.8\% sample (1140-min count). 
ANU-2258.

$5330 \pm 90$

Lake mud, 364-384 cm depth (1140-min count).

ANU-3596.

Lake mud, $389.5-409.5 \mathrm{~cm}$ depth. Dilution; 60\% sample (1100-min count).

ANU-2259.

$6360 \pm 100$

Lake mud, 501-521.5 cm depth. Dilution; 90\% sample (1040-min count).

\section{Core B56/78 Series}

Collected by D. Walker and J. L Neale 1978; submitted by D. Walker 1979.

ANU-3597.

Lake mud, $19-39 \mathrm{~cm}$ depth. Dilution; $71 \%$ sample (1080-min count).

ANU-2260.

$1400 \pm 80$

$\delta^{13} C=-25.3 \%$

$2780 \pm 90$

Lake mud, $147.5-163.5 \mathrm{~cm}$ depth. Dilution; $72 \%$ sample (1140-min count).

ANU-2261.

$4170 \pm 80$

Lake mud, $294.5-314.5 \mathrm{~cm}$ depth (1060-min count).

ANU-2262.

$4980 \pm 90$

Lake mud, $402.5-422.5 \mathrm{~cm}$ depth (1060-min count).

ANU-3598.

Lake mud, 427.5-447.5 cm depth. Dilution; 78\% sample (1100-min count).

$4810 \pm 100$

$\delta^{13} C=-26.8 \%$

ANU-2263.

$6570 \pm 90$

Lake mud, 538.5-58.5 cm depth (1060-min count).

\section{Core 58/78 Series}

Collected by D. Walker and J. L. Neale 1978; submitted by Y. Chen 1983.

ANU-3664.

Organic mud, $433-453 \mathrm{~cm}$ depth. Dilution; $83 \%$ sample (1000-min count).

ANU-3665.

Organic mud, $459-479 \mathrm{~cm}$ depth. Dilution; 87\% (1000-min count).

ANU-3667.

$7150 \pm 120$

Organic mud, $510-530 \mathrm{~cm}$ (1120-min count). 
ANU-3668.

$7440 \pm 100$

Organic mud, $547-567 \mathrm{~cm}$ depth (1000-min count).

$\delta^{13} C=-28.9 \%$

ANU-3669.

Organic mud, $571-591 \mathrm{~cm}$ depth (1120-min count).

$8040 \pm 120$

$\delta^{13} C=-27.0 \%$

\section{Core 63/78 Series}

Collected by D. Walker and J. L. Neale 1978; submitted by D. Walker 1979.

ANU-2264.

$2040 \pm 90$

Lake mud, 38.5-58.5 cm depth (960-min count).

ANU-2265.

$3770 \pm 90$

Lake mud, $172.2-192.2 \mathrm{~cm}$ depth (1100-min count).

ANU-3599.

Lake mud, $200.5-220.5 \mathrm{~cm}$ depth (1080-min count).

ANU-2266.

$4130 \pm 80$

$\delta^{13} C=-25.7 \%$

Lake mud, $290.5-310.5 \mathrm{~cm}$ depth (1320-min count).

ANU-3600.

Lake mud, $417.5-428.5 \mathrm{~cm}$ depth (1080-min count).

$8150 \pm 120$

$\delta^{13} C=-24.7 \%$

ANU-2267.

$8070 \pm 100$

Lake mud, $431.5-450 \mathrm{~cm}$ depth (1100-min count).

ANU-2268.

$9180 \pm 150$

Lake mud, 497-517 cm depth. Dilution; 79\% sample (1040-min count).

ANU-2269.

$9930 \pm 130$

Lake mud, $531.5-551.5 \mathrm{~cm}$ depth (1120-min count).

\section{Core 69/78 Series}

Collected by D. Walker and J. L. Neale 1978; submitted by D. Walker 1979.

ANU-2270.

$2020 \pm 80$

Lake mud, 35-55 cm depth (1120-min count).

ANU-3601.

$4350 \pm 90$

Lake mud, $149.5-169.5 \mathrm{~cm}$ depth (980-min count). 
ANU-3602.

Lake mud, $169.5-189.5 \mathrm{~cm}$ depth (1080-min count).

$5040 \pm 90$

$\delta^{13} C=-28.9 \%$

ANU-2271.

$7650 \pm 100$

Lake mud, 317.3-337.3 cm depth. Dilution; 87\% sample (1120-min count).

ANU-2272.

$9430 \pm 110$

Lake mud, $421.8-441.8 \mathrm{~cm}$ depth (1040-min count).

ANU-2273.

$10,600 \pm 110$

Lake mud, $470.8-490.8 \mathrm{~cm}$ depth (1740-min count).

\section{Core 379 Series}

ANU-2719 to -2721 collected by C. Constable, D. Walker and J. L. Neale 1979; submitted by D. Walker 1981; ANU-2717, -2718, -2722 and -2723 collected by C. Constable, D. Walker and J. L. Neale 1979; submitted by C. Constable 1981.

ANU-2717.

$2250 \pm 90$

Lake sediment, $1-17 \mathrm{~cm}$ depth. Dilution; 60\% sample (940-min count).

ANU-2718.

$3450 \pm 150$

Lake sediment, $159.5-179.5 \mathrm{~cm}$ depth. Dilution; 33\% sample (1020-min count).

ANU-2719.

$9110 \pm 100$

Lake sediment, $567.5-587.5 \mathrm{~cm}$ depth (1020-min count).

ANU-2720.

Lake sediment, $612.5-621.5 \mathrm{~cm}$ depth, and organic sediment, $621.5-632.5 \mathrm{~cm}$ depth. Two separate components.

ANU-2720A. Lake sediment. Dilution; 55\% sample (1020-min count).

ANU-2720B. Organic sediment. Dilution; 98\% sample (1360-min count).

$9360 \pm 150$

$9540 \pm 120$

ANU-2721.

$10,600 \pm 120$

Lake sediment, $644.5-664.5 \mathrm{~cm}$ depth (920-min count).

ANU-2722.

$17,300 \pm 550$

Lake sediment, $767.5-787.5 \mathrm{~cm}$ depth. Dilution; $41 \%$ sample (1260-min count).

ANU-2723.

$14,400 \pm 570$

Lake sediment, $880.5-90.5 \mathrm{~cm}$ depth. Dilution; $27 \%$ sample (1360-min count). 


\section{Core 479 Series}

Collected by C. Constable, D. Walker and J. L. Neale 1979; submitted by D. Walker 1981.

ANU-2724.

$3120 \pm 90$

Lake sediment, $105-125 \mathrm{~cm}$ depth. Dilution; 67\% sample (1140-min count).

ANU-2725.

$4060 \pm 90$

Lake sediment, 180-198.5 cm depth. Dilution; 71\% sample (1220-min count).

ANU-2726.

$6580 \pm 110$

Lake sediment, 251-269 cm depth. Dilution; 55\% sample (1120-min count).

ANU-2727.

$5100 \pm 80$

Lake sediment, $292.5-312.5 \mathrm{~cm}$ depth. Dilution; 83\% sample (1420-min count).

ANU-2728.

$6020 \pm 80$

Organic sediment, 371.7-391.7 cm depth. Dilution; 99\% sample (1000-min count).

ANU-2729.

$7250 \pm 110$

Organic sediment, 402.2-420.2 cm depth. Dilution; 79\% sample (1080-min count).

ANU-2730.

$8640 \pm 100$

Organic sediment, 530.2-550.2 cm depth (1160-min count).

ANU-2731.

$9260 \pm 110$

Organic mud, 550.2-567.5 cm depth (960-min count)

ANU-2732.

$10,250 \pm 110$

Organic mud, 579.2-599.7 cm depth (1160-min count).

ANU-2733.

$10,150 \pm 110$

Organic mud, 611.2-630.2 cm depth (1120-min count).

ANU-2734.

$13,050 \pm 400$

Organic sediment, $759.5-779.5 \mathrm{~cm}$ depth. Dilution; $26 \%$ sample (1060-min count).

ANU-2735.

$13,600 \pm 170$

Organic mud, 910.7-930.7 cm depth (1800-min count). 


\section{Core 579 Series}

ANU-2445 to -2450 collected by C. Constable and J. L. Neale 1979; submitted by C. Constable 1980; ANU-2451 to -2456 collected by C. Constable and J. L. Neale 1979; submitted by D. Walker 1980.

ANU-2445.

$3930 \pm 140$

Lake sediment, 80-92 cm depth. Dilution; 34\% sample (1020-min count).

ANU-2446.

$4950 \pm 120$

Lake sediment, $147.6-167.6 \mathrm{~cm}$ depth. Dilution; $49 \%$ sample (980-min count).

ANU-2451.

$5990 \pm 90$

Lake mud, $201.9-214.9 \mathrm{~cm}$ depth (1080-min count).

ANU-2452.

$6360 \pm 90$

Lake mud, 221.9-235.4 cm depth.

ANU-2447.

$7360 \pm 100$

Lake sediment, 335.1-355.1 cm depth. Dilution; 88\% sample (1020-min count).

ANU-2453.

$7490 \pm 80$

Lake mud, $446.2-467 \mathrm{~cm}$ depth (1700-min count).

ANU-2454.

$8890 \pm 120$

Lake mud, 499-519 cm depth. Dilution; 73\% sample (1080-min count).

ANU-2448.

$12,600 \pm 290$

Lake sediment, 535-554.5 cm depth. Dilution; 31\% (1640-min count).

ANU-2449.

$10,800 \pm 240$

Lake sediment, $663.5-683.5 \mathrm{~cm}$ depth. Dilution; $40 \%$ sample (860-min count).

ANU-2455.

$11,800 \pm 260$

Lake mud, 732.1-767.1 cm depth. Dilution; 37\% sample (1000-min count).

ANU-2450.

$13,350 \pm 210$

Lake sediment, $940-957.5 \mathrm{~cm}$ depth. Dilution; 63\% sample (980-min count).

ANU-2456.

$11,350 \pm 400$

Lake mud, 1034.5-1054.5 cm depth. Dilution; 21\% sample (1040-min count). 


\section{Core B779 Series}

Collected by D. Walker and J. L. Neale 1979; submitted by C. Constable 1980.

ANU-2405.

$2820 \pm 130$

Mud and clay, 64-82 cm depth. Dilution; 31\% sample (1040-min count).

ANU-2406.

$2770 \pm 130$

Mud and clay, 93-112.5 cm depth. Dilution; $31 \%$ sample (1000-min count).

ANU-2408.

$3930 \pm 120$

Mud and clay, 190.5-207 cm depth. Dilution; 41\% sample (1000-min count).

ANU-2409.

$2540 \pm 130$

Mud and clay, $207-226.2 \mathrm{~cm}$ depth. Dilution; $12 \%$ sample (4040-min count).

ANU-2410.

$4830 \pm 1390$

Mud and clay, $275.9-293.7 \mathrm{~cm}$ depth. Dilution; 61\% sample (1000-min count).

ANU-2411.

$5890 \pm 90$

Mud and clay, 343.7-357.7 cm depth. Dilution; 79\% (1000-min count).

ANU-2412.

$9890 \pm 130$

Mud and clay, $521.6-531.6 \mathrm{~cm}$ depth (1000-min count).

ANU-2413.

$12,650 \pm 170$

Mud and clay, 577.1-597.1 cm depth. Dilution; 75\% sample (1020-min count).

ANU-2414.

$11,300 \pm 130$

Mud and clay, 597.1-617.1 cm depth. Dilution; 85\% sample (1080-min count).

ANU-2415.

$12,500 \pm 340$

Mud and clay, 697.6-716.1 cm depth. Dilution; 38\% sample (1000-min count).

ANU-2416.

$11,400 \pm 190$

Mud and clay, 958.9-978.9 cm depth. Dilution; 49\% sample (1080-min count).

\section{Core 83/1c Series}

Collected by D. Walker and J. L. Neale 1983; submitted by D. Walker 1987.

ANU-5880.

$2030 \pm 210$

Lake sediment, 1-3.8 cm depth. Dilution; 9\% sample (1972-min count). 
ANU-5881.

$2850 \pm 220$

Lake sediment, 91.2-94.1 cm depth. Dilution; 8\% sample (1972-min count).

ANU-5882.

$3440 \pm 270$

Lake sediment, 194.9-197.8 cm depth. Dilution; 6\% sample (2959-min count).

ANU-5883.

$5090 \pm 170$

Lake sediment, 310.4-313.4 cm depth. Dilution; 12\% sample (4438-min count).

ANU-5884.

$4530 \pm 200$

Lake sediment, 313.4-316.4 cm depth. Dilution; $12 \%$ sample (1972-min count).

ANU-5885.

$6430 \pm 140$

Lake sediment, 399.9-403.9 cm depth. Dilution; 21\% sample (2958-min count).

ANU-5886.

$7400 \pm 160$

Lake sediment, 499.4-502.4 cm depth. Dilution; 20\% sample (2465-min count).

ANU-6154.

$4650 \pm 320$

Lake sediment, 316.4-31.9.4 cm depth. Dilution; 12\% sample (3600-min count).

ANU-6155.

$4530 \pm 380$

Lake sediment, 309.4-31.0.4 cm depth. Dilution; 16\% sample (2958-min count).

ANU-6156.

$4150 \pm 220$

Lake sediment, 271.9-274.9 cm depth. Dilution; 7\% sample (2465-min count).

ANU-6157.

$4040 \pm 240$

Lake sediment, 204.4-207.4 cm depth. Dilution; 15\% sample (3600-min count).

ANU-6158.

$3700 \pm 470$

Lake sediment, 200.5-203.4 cm depth. Dilution; 7\% sample (2760-min count).

ANU-6159.

$3050 \pm 380$

Lake sediment, $166.4-169 \mathrm{~cm}$ depth. Dilution; 8\% sample (2730-min count).

ANU-6160.

$2820 \pm 220$

Lake sediment, 69.1-72 cm depth. Dilution; 7\% sample (1972-min count).

ANU-6161.

$2510 \pm 250$

Lake sediment, 53-56 crn depth. Dilution; 5\% sample (1972-min count). 
ANU-6499.

Lake sediment, $76.2-82 \mathrm{~cm}$ depth. Dilution; $17 \%$ sample (2820-min count).

ANU-6500.

$3370 \pm 110$

Lake sediment, 139.2-145 cm depth. Dilution; 19\% sample (2959-min count).

ANU-6501.

$3780 \pm 150$

Lake sediment, 213.4-218.4 cm depth. Dilution; 11\% sample (2959-min count).

ANU-6502.

$6410 \pm 170$

Lake sediment, 423.4-429.4 cm depth. Dilution; 38\% sample (2010-min count).

ANU-6606.

$3550 \pm 220$

Lake sediment, 118-124 cm depth. Dilution; $17 \%$ sample (2880-min count).

Est. $\delta^{13} C=-16.0 \%$

ANU-6607.

$5510 \pm 180$

Lake sediment, 367.4-373.4 cm depth. Dilution; $29 \%$ sample (2850-min count).

ANU-6608.

$6430 \pm 150$

Lake sediment, $437-443 \mathrm{~cm}$ depth. Dilution; $40 \%$ sample (2820-min count).

ANU-6856.

$4240 \pm 210$

Lake sediment, $42-48 \mathrm{~cm}$ depth. Dilution; $60 \%$ sample (2959-min count).

$\delta^{13} C=-30.3 \%$

ANU-6857.

$2580 \pm 150$

Lake sediment, 156-159 cm depth. Dilution; 10\% sample (1973-min count).

Collected 1983 and submitted 1991 by D. Walker.

ANU-7972.

$2550 \pm 190$

Lake mud, 30-36 cm depth. Dilution; 63\% sample (2959-min count).

$\delta^{13} C=-30.4 \%$

ANU-7973.

Lake mud, $140-150 \mathrm{~cm}$ depth (2959-min count).

$3200 \pm 170$

$\delta^{13} C=-30.9 \%$

ANU-7974.

$3460 \pm 140$

Lake mud, $180-192 \mathrm{~cm}$ depth. Dilution; $28 \%$ sample (3000-min count).

ANU-7975.

$4800 \pm 200$

Lake mud, 338-341 cm depth. Dilution; 73\% sample (2959-min count).

$\delta^{13} C=-30.5 \%$ 
ANU-8066.

Lake mud, 14-20 cm depth. Dilution; 25\% sample (2959-min count).

ANU-8067.

Lake mud, 90-95 cm depth. Dilution; $14 \%$ sample (2959-min count).

ANU-8068.

Lake mud, 205-218 cm depth. Dilution; 82\% sample (2959-min count).

ANU-8069.

Lake mud, 219-222 cm depth. Dilution; 49\% sample (2959-min count).

ANU-8071.

Lake mud, 325-331 cm depth. Dilution; 77\% sample (1973-min count).

ANU-8072.

Lake mud, $412-418 \mathrm{~cm}$ depth. Dilution; $26 \%$ sample (2959-min count).

ANU-8125.

Lake mud, $258-270 \mathrm{~cm}$ depth (2959-min count).

ANU-8126.

Lake mud, $430-440 \mathrm{~cm}$ depth. Dilution; $24 \%$ sample (2850-min count).
$1620 \pm 270$

Est. $\delta^{13} \mathrm{C}=-30.0 \%$

$1430 \pm 350$

Est. $\delta^{13} C=-30.0 \%$

$4090 \pm 190$

Est. $\delta^{13} \mathrm{C}=-30.0 \%$

$3940 \pm 230$

Est. $\delta^{13} C=-30.0 \%$

$4610 \pm 200$

Est. $\delta^{13} C=-30.0 \%$

$4670 \pm 90$

$3910 \pm 180$

$\delta^{13} C=-30.0 \%$

$5230 \pm 190$

Est. $\delta^{13} C=-30.0 \%$

\section{Core 83/9 Series}

Collected by D. Walker and J. L. Neale 1983; submitted by D. Walker (5622-5632) 1986, (6604 6605) 1988 and Y. Chen (4464-4471, 4024-4030) 1984

ANU-5622.

$1640 \pm 130$

Lake sediment, $1-4 \mathrm{~cm}$ depth. Dilution; $10 \%$ sample (3452-min count).

ANU-6604.

$2240 \pm 120$

Lake sediment, $28-32 \mathrm{~cm}$ depth. Dilution; $14 \%$ sample (2958-min count).

ANU-5623.

$2440 \pm 160$

Lake sediment, 51-54 cm depth. Dilution; 8\% sample (2959-min count).

ANU-5624.

$2980 \pm 200$

Lake sediment, 55-58 cm depth. Dilution; 7\% sample (2465-min count). 
ANU-5625.

Lake sediment, 93.5-96.5 cm depth. Dilution; 14\% sample (2904-min count).

ANU-6605.

$3630 \pm 90$

Lake sediment, $96.5-101.5 \mathrm{~cm}$ depth. Dilution; $29 \%$ sample (2958-min count).

ANU-5626.

$3550 \pm 150$

Lake sediment, $104.5-107.5 \mathrm{~cm}$ depth. Dilution; $10 \%$ sample (2959-min count).

ANU-5627.

$4260 \pm 160$

Lake sediment, $148.5-151.5 \mathrm{~cm}$ depth. Dilution; $15 \%$ sample (1972-min count).

ANU-5628.

$4250 \pm 160$

Lake sediment, $153.5-156.5 \mathrm{~cm}$ depth. Dilution; $13 \%$ sample (1972-min count).

ANU-5629.

$4960 \pm 160$

Lake sediment, 191-194 cm depth. Dilution; 14\% sample (2958-min count).

ANU-5630.

$4820 \pm 210$

Lake sediment, 194-197 cm depth. Dilution; 10\% sample (2465-min count).

ANU-5631.

$5340 \pm 150$

Lake sediment, 220-223 cm depth. Dilution; $17 \%$ sample (2958-min count).

ANU-5632.

$5820 \pm 150$

Lake sediment, 252-255 cm depth. Dilution; $15 \%$ sample (2959-min count).

ANU-4024.

Lake mud, $283-288 \mathrm{~cm}$ depth. Dilution; $28 \%$ sample (1400-min count).

$5630 \pm 160$

$\delta^{13} C=-31.0 \%$

ANU-4464.

$6640 \pm 160$

Lake mud, 332.6-337.6 cm depth. Dilution; 31\% sample (1380-min count).

$\delta^{13} C=-27.8 \%$

ANU-4025.

$6520 \pm 150$

Lake mud, $381.2-386.2 \mathrm{~cm}$ depth. Dilution; $22 \%$ sample.

$\delta^{13} C=-30.2 \%$

ANU-4465.

$6590 \pm 220$

Lake mud, 405.6-410.6 cm depth. Dilution; 31\% sample (1320-min count).

$\delta^{13} C=-28.4 \%$

ANU-4466.

$7100 \pm 250$

Lake mud, $430-435 \mathrm{~cm}$ depth. Dilution; $35 \%$ sample (1080-min count).

$\delta^{13} C=-24.0 \%$ 
ANU-4467.

Lake mud, $455-460 \mathrm{~cm}$ depth. Dilution; 32\% sample (1080 count time)

ANU-4468.

Lake mud, $479.3-484.3 \mathrm{~cm}$ depth. Dilution; 39\% sample (1360-min count).

ANU-4469.

Lake mud, 498.5-503.5 cm depth. Dilution; 40\% sample (1120-min count).

\section{ANU-4470.}

Lake mud, 530-535 cm depth. Dilution; 66\% sample (1120-min count).

ANU-4471.

Lake mud, 548-553 cm depth. Dilution; 56\% sample (1000-min count).

ANU-4026.

Lake mud, 558-563 cm depth. Dilution; 28\% sample (940-min count).

ANU-4027.

Lake mud, 568-573 cm depth. Dilution; 37\% sample (960-min count).

ANU-4028.

Lake mud, 985-995 cm depth. Dilution; 25\% sample (2100-min count).

ANU-4029.

Lake mud, 1000-1010 cm depth. Dilution; 22\% sample (1000-min count).

ANU-4030.

Lake mud, $1180-1190 \mathrm{~cm}$ depth. Dilution; $18 \%$ sample.
$5740 \pm 200$

$\delta^{13} C=-25.1 \%$

$8630 \pm 210$

$\delta^{13} C=-27.2 \%$

$8020 \pm 210$

$\delta^{13} \mathrm{C}=-28.0 \%$

$8740 \pm 160$

$\delta^{13} C=-26.7 \%$

$9480 \pm 160$

$\delta^{13} C=-31.3 \%$

$9960 \pm 290$

$\delta^{13} C=-26.1 \%$

$11,160 \pm 260$

$\delta^{13} C=-24.1 \%$

$13,440 \pm 300$

$\delta^{13} C=-21.7 \%$

$11,240 \pm 380$

$\delta^{13} C=-20.2 \%$

$11,570 \pm 420$

$\delta^{13} C=-20.2 \%$

Barrine-Core Samples from Water Depth Presently $\leq 50$ m (Table 3)

TABLE 3. Barrine Water (presently $\leq 50 \mathrm{~m}$ )

\begin{tabular}{lll}
\hline Depth (m) & Age (ka BP) & \multicolumn{1}{c}{ Sediment } \\
\hline $0-3.7$ & $0-5.5$ & Unlaminated mud, variously clayey \\
$3.7-3.8$ & & Slip structures \\
$3.8-4.6$ & & Sandy clay \\
$4.6-5.9$ & $8-10$ & Compact silty clay mud \\
\hline
\end{tabular}

Core 68/78 Series

Collected by D. Walker and J. L. Neale 1978; submitted by Y. Chen 1983. 
ANU-3671.

Organic mud, 190-210 cm depth. Dilution; 81\% (1000-min count).

ANU-3672.

Organic mud, 300-320 cm depth. (940-min count).

ANU-3673.

Organic mud, $420-440 \mathrm{~cm}$ depth. Dilution; 80\% (1000-min count).

\section{ANU-3675.}

Organic mud, 540-560 cm depth (1000-min count).

\section{Core 71/78 Series}

Collected by D. Walker and J. L. Neale 1978; submitted by Y. Chen 1983.

\section{ANU-3655.}

Organic mud, 50-70 cm depth. Dilution; 87\% sample (1000-min count).

ANU-3656.

Organic mud, 179-199 cm depth. Dilution; 52\% sample (1000-min count).

ANU-3657.

Organic mud, 240-260 cm depth. Dilution; 77\% sample (980-min count).

ANU-3658.

Organic mud, $439-459 \mathrm{~cm}$ depth. Dilution; $71 \%$ sample (1000-min count).

ANU-3659.

Organic mud, 471-491 cm depth (1020-min count).

\section{Core B75/78 Series}

Collected by D. Walker and J. L. Neale 1978; submitted by D. Walker 1979.

ANU-2300.

$2180 \pm 70$

Lake mud, 64-84 cm depth. Dilution; $66 \%$ sample (2480-min count).

ANU-2301.

$3070 \pm 80$

Lake mud, $138-158 \mathrm{~cm}$ depth. Dilution; $85 \%$ sample (1400-min count).

ANU-2302.

$4880 \pm 80$
$8^{13} C=-31.8 \%$

$6480 \pm 100$

$\delta^{13} \mathrm{C}=-28.9 \%$

$9150 \pm 130$

$\delta^{13} C=-27.7 \%$

$10,090 \pm 120$

$\delta^{13} C=-28.0 \%$

$\delta^{13} C=-22.7 \%$

$850 \pm 90$

$2050 \pm 100$

$\delta^{13} C=-28.6 \%$

$3730 \pm 90$

$\delta^{13} C=-28.7 \%$

$4110 \pm 80$

$\delta^{13} C=-29.2 \%$

Lake mud, $246.5-266.5 \mathrm{~cm}$ depth (1300-min count). 
ANU-2303.

$5880 \pm 90$

Lake mud, 314-334 cm depth (1160-min count).

\section{Core 83/7A Series}

Collected by J. L. Neale and Y. Chen 1983; submitted by Y. Chen 1984.

ANU-3899.

Organic mud, 300-320 cm depth. Dilution; 68\% (1080-min count).

ANU-3900.

Organic mud, $360-380 \mathrm{~cm}$ depth (920-min count).

ANU-4461.

Lake mud, $390-410 \mathrm{~cm}$ depth. Dilution; $80 \%$ sample (1340-min count).

ANU-4462.

Lake mud, $450-470 \mathrm{~cm}$ depth. Dilution; $93 \%$ sample (1060-min count).

ANU-4463.

Lake mud, $500-520 \mathrm{~cm}$ depth. Dilution; 79\% sample (1120-min count).
$3500 \pm 200$

$\delta^{13} C=-28.9 \%$

$4700 \pm 200$

$\delta^{13} C=-29.9 \%$

$5200 \pm 80$

$\delta^{13} C=-25.5 \%$

$5940 \pm 90$

$\delta^{13} C=-26.3 \%$

$7630 \pm 100$

$\delta^{13} C=-24.7 \%$

Barrine-Frozen Samples from Water Depth Presently $67 \mathrm{~m}$

Samples of uppermost very unconsolidated mud collected by in-situ freezing at Point 1 , lake center.

Frozen Fingers. Collected by D. Walker and J. L. Neale 1979; submitted by D. Walker 1983.

ANU-3618.

Lake sediment, 0-15 cm depth. Dilution; 64\% sample (1380-min count).

ANU-3619.

Lake sediment, $15-21 \mathrm{~cm}$. Dilution; 61\% sample (1080-min count).

ANU-3620.

Lake sediment, 21-26.5 cm depth. Dilution; 69\% sample (1100-min count).
$98.2 \pm 1.2 \mathrm{pMC}$ $\delta^{13} C=-25.2 \%$

$99.0 \pm 1.0 \mathrm{pMC}$ $\delta^{13} \mathrm{C}=-27.0 \%$

$101.1 \pm 1.0 \mathrm{pMC}$ $\delta^{13} C=-31.2 \%$

Comment: Depths are from apparent mud-water interface. Similar levels were bulked to attain adequate sample size. Excess water was removed by centrifugation and the samples were oven-dried.

Frozen Slabs. Collected by D. Walker and J. Neale 1986 and 1987; submitted by D. Walker 1989.

ANU-6964.

$103.0 \pm 3.2 \mathrm{pMC}$

Lake mud, 0-5 cm depth. Dilution; 24\% (2959-min count). 
ANU-6965.

$98.2 \pm 1.2 \mathrm{pMC}$

Lake mud, 5-10 cm depth. Dilution; 15\% sample (2764-min count).

ANU-6966.

$640 \pm 90$

Lake mud, $10-15 \mathrm{~cm}$ depth. Dilution; $17 \%$ sample (2959-min count).

ANU-6967.

$260 \pm 80$

Lake mud, $15-20 \mathrm{~cm}$ depth. Dilution; $20 \%$ sample (2959-min count).

ANU-6968.

$450 \pm 170$

Lake mud, 20-25 cm depth. Dilution; $80 \%$ sample (2959-min count).

ANU-6969.

$99.1 \pm 1.0 \mathrm{pMC}$

Lake mud, $25-30 \mathrm{~cm}$ depth. Dilution; $21 \%$ sample (2959-min count).

Comment: Depths are from mud-water interface.

Frozen Slabs. Collected by D. Walker and J. Neale 1987; submitted by D. Walker 1991.

ANU-7968.

Lake mud, 50-55 cm depth. Dilution; 55\% sample (3240-min count).

ANU-7969.

Lake mud, $60-65 \mathrm{~cm}$ depth. Dilution; $26 \%$ sample (3210-min count).

ANU-7970.

Lake mud, 70-75 cm depth. Dilution; 62\% sample (1973-min count).

ANU-7971.

Lake mud, 80-85 cm depth. Dilution, $44 \%$ sample (2959-min count).

ANU-8111.

Lake mud, 19-24 cm depth. Dilution; 25\% sample (2959-min count).

ANU-8112.

Lake mud, 29-34 cm depth. Dilution; 45\% (2959-min count).

ANU-8113.

Lake mud, 90-95 cm depth (2959-min count).

ANU-8114.

Lake mud, 39-44 cm depth. Dilution; $42 \%$ sample (2466-min count).
$650 \pm 80$

$\delta^{13} C=-28.8 \%$

$630 \pm 110$

$\delta^{13} C=-30.9 \%$

$1050 \pm 210$

$\delta^{13} \mathrm{C}=-30.3 \%$

$1080 \pm 60$

$\delta^{13} C=-29.6 \%$

$230 \pm 70$

$\delta^{13} C=-31.7 \%$

$430 \pm 50$

$\delta^{13} C=-30.5 \%$

$1240 \pm 160$

$\delta^{13} C=-31.0 \%$

$490 \pm 70$ 
Comment: Depths corrected to distances below reference lamination $(\sim 20 \mathrm{~cm}$ below mud-water interface).

Barrine-Contemporary Sedimentation

1. Collected by D. Walker and J. L. Neale 1979; submitted by D. Walker 1980.

ANU-2545.

$134.5 \pm 7.3 \mathrm{pMC}$

$\delta^{13} \mathrm{C}=-27.3 \%$

Leaf litter from water's edge, about to enter the lake. Dilution; $91 \%$ sample (1080-min count).

Comment: No pretreatment.

ANU-2546.

$980 \pm 50$

$\delta^{13} \mathrm{C}=-29.5 \%$

Lake mud, upper 5-10 cm of thick unconsolidated mud at sediment interface, beneath $65 \mathrm{~m}$ of water at center of lake (1080-min count).

2. Collected and submitted by D. Walker 1983 .

ANU-3615.

Twigs and wood fragments, coarse fraction $(>2 \mathrm{~mm})(1000-\mathrm{min}$ count).

ANU-3616.
$110.0 \pm 0.9 \mathrm{pMC}$

$\delta^{13} C=-24.2 \%$

Woody material, medium fraction $(0.25-2 \mathrm{~mm})$. Dilution; $95 \%$ sample (1300-min count).

ANU-3617.

Woody material, fine fraction ( $<0.25 \mathrm{~mm})(1320$-min count).
$122.3 \pm 0.9 \mathrm{pMC}$

$\delta^{13} \mathrm{C}=-25.4 \%$

Comment: ANU-3615 to -3617 are from the same grab sample (Z1) under $1 \mathrm{~m}$ of water at the northwest lake margin. No pretreatment.

3. Grab samples of mud-water interface. Collected and submitted by D. Walker 1990.

ANU-7412.

$98.4 \pm 1.1 \mathrm{pMC}$

Slurpy mud, surface deposit Point 1, below $67 \mathrm{~m}$ water. Dilution; 83\% sample (3000-min count).

ANU-7413.

$100.2 \pm 1.1 \mathrm{pMC}$

Slurpy mud, surface deposit Point 6, below $42 \mathrm{~m}$ water. Dilution; 89\% sample (3000-min count).

ANU-7414.

$97.8 \pm 1.3 \mathrm{pMC}$

Slurpy mud, surface deposit Point 9, below $63 \mathrm{~m}$ water. Dilution; 40\% sample (3000-min count).

Comment: ANU-7412 to -.7414 had excess water removed by centrifugation and were then ovendried. 
Eacham-Core Samples from Water Depth Presently $\sim 64 m$

\section{Core E1/77 Series}

Collected by D. Walker and J. L. Neale 1977; submitted by C. Constable 1982.

ANU-3169.

$320 \pm 100$

Lake sediment, 0-18 cm depth (1100-min count).

ANU-3170.

$4990 \pm 80$

Lake sediment, 488-508 cm depth (1220-min count).

\section{Core E2/77 Series}

Collected by D. Walker and J. L. Neale 1977; submitted by C. Constable 1981.

ANU-3016.

$1470 \pm 100$

Lake sediment, $75-93 \mathrm{~cm}$ depth (2500-min count).

ANU-3017.

$3020 \pm 80$

Lake sediment, 316-336 cm depth (1100-min count).

ANU-3018.

$4480 \pm 100$

Lake sediment, 498.3-518.3 cm depth. Dilution; 91\% sample (1040-min count).

\section{Core E77/78 Series}

Collected by D. Walker and J. L. Neale 1978, submitted by C. Constable 1981.

ANU-3171.

$1230 \pm 70$

Lake sediment, $118-137 \mathrm{~cm}$ depth. (1580-min count).

ANU-3172.

$1690 \pm 150$

Lake sediment, $214-233 \mathrm{~cm}$ depth. (1100-min count).

ANU-3173.

Lake sediment, $311-331 \mathrm{~cm}$ depth. (1200-min count).

ANU-3174.

$3660 \pm 80$

Lake sediment, $448-461 \mathrm{~cm}$ depth. (1320-min count).

\section{Core E78/78 Series}

Collected by D. Walker and J. L. Neale 1978; submitted by D. Walker and M. Goodfield 1983. 


\section{ANU-3532.}

Lake mud, $30-50 \mathrm{~cm}$ depth.

ANU-3532A.

$590 \pm 240$

Coarse fraction $(>250 \mu)$. Dilution; $12 \%$ sample (1460-min count).

ANU-3532B.

$400 \pm 120$

Fine fraction $(<250 \mu)$. Dilution; $29 \%$ sample $(1000-\min$ count).

ANU-3533.

$1860 \pm 70$

Lake mud, 238-258 cm depth (1500-min count).

ANU-3534.

$2060 \pm 70$

Lake mud, 256-276 cm depth (1100-min count).

ANU-3537.

$3200 \pm 80$

Lake mud, $406-426 \mathrm{~cm}$ depth. Fine fraction $(<250 \mu)(1020-\mathrm{min}$ count $)$.

$\delta^{13} C=-26.1 \%$

ANU-3538.

$3510 \pm 80$

Lake mud, $431-451 \mathrm{~cm}$ depth. Fine fraction $(<250 \mu)(1000-\mathrm{min}$ count).

ANU-3535.

$4470 \pm 80$

Lake mud, 524-534 cm depth (1000-min count).

\section{ANU-3536.}

$3930 \pm 90$

$\delta^{13} \mathrm{C}=-26.1 \%$

Lake mud, $539-549 \mathrm{~cm}$ depth. Fine fraction $(<250 \mu)$. Dilution; $94 \%$ sample (1020-min count).

Comment: ANU-3532A, -3536 to -3538 were macerated lightly, wet sieved, and then given pretreatment $\mathrm{A} ; \mathrm{ANU}-3532 \mathrm{~B}$ was given pretreatment $\mathrm{B}(\mathrm{i})$.

\section{Core E80/78 Series}

Collected by D. Walker and J. L. Neale 1978; submitted by C. Constable 1981.

ANU-3019.

$1540 \pm 90$

Lake sediment, $107-127 \mathrm{~cm}$ depth (1280-min count).

ANU-3020.

$4640 \pm 80$

Lake sediment, $537.5-557.5 \mathrm{~cm}$ depth (1220-min count).

\section{Core 83/23A Series}

Collected by D. Walker, J. L. Neale and Y. Chen 1983; submitted by Y. Chen 1984. 
ANU-4031.

$4210 \pm 130$

Lake mud, $475-480 \mathrm{~cm}$ depth. Dilution; $41 \%$ sample (1000-min count).

ANU-4032.

$6260 \pm 160$

Lake mud, 790-795 cm depth. Dilution; 36\% sample (1100-min count).

$\delta^{13} C=-30.7 \%$

ANU-4033.

$9130 \pm 190$

Lake mud, $970-975 \mathrm{~cm}$ depth. Dilution; $25 \%$ sample.

ANU-4034.

$8980 \pm 190$

Lake mud, $975-980 \mathrm{~cm}$ depth. Dilution; $42 \%$ sample (1000-min count).

$\delta^{13} C=-28.6 \%$

\section{Eacham-Contemporary Sedimentation}

Collected and submitted by D. Walker 1977.

ANU-2046.

Leaves (Ficus), from ground surface beside lake (2100-min count).

$138.2 \pm 1.0 \mathrm{pMC}$

Est. $\delta^{13} \mathrm{C}=-27.0 \%$

ANU-2047.

$140.7 \pm 1.1 \mathrm{pMC}$

Leaves (mixed), from ground surface beside lake (3440-min count).

ANU-2048.

$136.6 \pm 1.0 \mathrm{pMC}$

Leaves (Acacia), from ground surface beside lake (1420-min count).

ANU-2049.

$142.2 \pm 1.1 \mathrm{pMC}$

Leaves (Pandanus), from surface beside lake (3480-min count).

ANU-2050.

$142.5 \pm 1.1 \mathrm{pMC}$

Leaves (Araucaria), from plantation on lake edge (3420-min count).

Comment: No pretreatment for this series.

\section{REFERENCES}

Chen, Y. (ms.) 1986 Early Holocene vegetation dynamics of Lake Barrine basin, northeast Queensland, Australia. Ph.D. dissertation, Department of Biogeography and Geomorphology, The Australian National University, Canberra, Australia: 246 p.

1987 Pollen and sediment distribution in a small crater lake in northeast Queensland, Australia. Pollen et Spores 29: 89-110.

1988 Early Holocene population expansion of some rainforest trees at Lake Barrine basin, Queensland. Australian Journal of Ecology 13: 225-233.
Currie, L. A. and Polach, H. A. 1980 Exploratory analysis of the international radiocarbon cross-calibration data: Consensus values and interlaboratory error; preliminary note. In Stuiver, M. and Kra, R. S., eds., Proceedings of the 10th International Radiocarbon Conference. Radiocarbon 22(3): 933-935.

Goodfield, M. L. (ms.) 1983 A Holocene pollen analytical study of Lake Eacham, Atherton Tableland, northeast Queensland. B.Sc. (Hons.) dissertation, Department of Geography, Monash University, Melbourne, Australia: $147 \mathrm{p}$. 
Green, D. G. and Dolman, G. S. 1988 Fine resolution pollen analysis. Journal of Biogeography 15: 685701.

Grindrod, J. F. (ms.) 1979 Frozen sediments from a tropical lake: A palaeoecological study of Lake Eacham, north-east Queensland. B.A. (Hons.) dissertation, Department of Geography, Monash University, Melbourne, Australia: $108 \mathrm{p}$.

Polach, H. A. 1979 Correlation of ${ }^{14} \mathrm{C}$ activity of NBS oxalic acid with Arizona 1850 wood and ANU sucrose standards. In Berger, Rainer and Suess, H. E., eds., Radiocarbon Dating. Proceedings of the 9th International ${ }^{14} \mathrm{C}$ Conference. Berkeley, University of California Press: 115-124.
Timms, B. V. 1976 Morphology of Lakes Barrine, Eacham and Euramoo, Atherton Tableland, north Queensland. Proceedings of the Royal Society of Queensland 87: 81-84.

1979 The benthos of some lakes in northeastern Queensland. Proceedings of the Royal Society of Queensland 90: 57-64.

Walker, D. 1988 (ms.) The Lake Barrine Project. In 7th International Palynological Congress Excursion LB6 Guide. Vegetation, Historical Plant Geography and Late Cenozoic Vegetation History of the Northeast Queensland Humid Tropics. Department of Biogeography and Geomorphology, The Australian National University: 38-46. 